\title{
Induced parity violating thermal effective action for $2+1$-dimensional fermions interacting with a non-Abelian background
}

\author{
F. T. Brandt ${ }^{a}$, Ashok Das $^{b}$ and J. Frenkel ${ }^{a}$ \\ ${ }^{a}$ Instituto de Física, Universidade de São Paulo \\ São Paulo, SP 05315-970, BRAZIL \\ ${ }^{b}$ Department of Physics and Astronomy \\ University of Rochester \\ Rochester, NY 14627-0171, USA
}

October 27, 2018

\begin{abstract}
We study the parity breaking effective action in $2+1$ dimensions, generated, at finite temperature, by massive fermions interacting with a non-Abelian gauge background. We explicitly calculate, in the static limit, parity violating amplitudes up to the seven point function, which allows us to determine the corresponding effective actions. There are two classes of such actions that arise, namely, terms that do not manifestly depend on $\vec{E}$ and ones that do. We derive the exact effective action that is not manifestly dependent on $\vec{E}$. For the other class that depends explicitly on $\vec{E}$, there are families of terms that can be determined order by order in perturbation theory. We attempt to generalize our results to non-static backgrounds through the use of time ordered exponentials and prove gauge invariance, both small and large, of the resulting effective action. We also point out some open questions that need to be further understood.
\end{abstract}




\section{Introduction:}

Chern-Simons theories in $2+1$ dimensions [1], 2] have attracted a lot of attention in the past few years for a variety of reasons [3]. One of the issues studied extensively, for example, is the question of large gauge invariance at finite temperature [4]-12]. In particular, it is known for sometime now that, at finite temperature, massive fermions interacting with a background nonAbelian gauge field induce a Chern-Simons term with a coefficient which is a continuous function of temperature [ [1]. Therefore, at an arbitrary temperature, the Chern-Simons coefficient cannot have a discrete value as would be necessary for invariance under large gauge transformations.

There has been a lot of progress in understanding this puzzle in the past few years. It has been shown, for example, within the context of $0+1$ dimensional fermions interacting with an external Abelian gauge field, that at finite temperature, an infinity of terms is induced in the effective action [5] in such a way that large gauge invariance is restored in the complete effective action, even though at any finite order in perturbation theory such an invariance will not be manifest [5, 10]. Subsequently, this analysis has been generalized to $2+1$ dimensional fermions interacting with an Abelian gauge background [6]-9]. Although, at zero temperature, Abelian gauge transformations correspond to transformations with a trivial winding number and impose no restrictions on the Chern-Simons coefficient, at finite temperature, because of periodicity (in the imaginary time formalism), Abelian transformations with nontrivial winding are possible. Furthermore, at finite temperature, amplitudes and, therefore, the effective action become non-analytic functions at the origin in the energy-momentum plane [13-15] (because of additional channels available for processes in a thermal medium). In the case of Abelian gauge backgrounds, we understand the resolution of the puzzle of large gauge invariance, at least, in two distinct conventional limits. In the long wave (LW) limit, where all spatial momenta vanish, it has been shown through explicit perturbative calculations up to the four point function that large gauge invariance is not a problem order by order in perturbation theory [16]. On the other hand, in the static limit, where all energies vanish, at any order in perturbation, large gauge invariance is not manifest. However, it is also known that one can sum the leading order terms in the parity violating effective action in this limit and the resulting effective action has a form which is a generalization of the $0+1$ dimensional result and is invariant under large gauge transformations much the same way [6]-9]. The leading 
order parity violating effective action, in this limit, also corresponds to the exact effective action (parity violating) when the electric field vanishes. Beyond the leading order, one picks up contributions which are proportional to the electric field and all such terms are manifestly large gauge invariant [17. It is worth pointing out here that, for any intermediate limit (between the two conventional limits, namely, LW and static), calculations become extremely complicated owing to the analytic continuation that is needed in the imaginary time formalism. As a consequence, explicit forms for the parity violating effective action are not available in this regime. However, it is natural to believe that large gauge invariance will hold in the complete effective action in this case as well.

The studies in the Abelian gauge background have given us very valuable insights into the question of large gauge invariance in such theories. With this knowledge, therefore, it is natural to reanalyze the question of large gauge invariance for a $2+1$ dimensional massive fermion interacting with a nonAbelian gauge background at finite temperature (which, in fact, led to all these studies). We would like to point out that such an analysis was, in fact, carried out earlier [9], in the path integral formalism, for a very restrictive gauge background of the form (gauge potentials are matrices belonging to $S U(N))$

$$
\begin{aligned}
A_{i}=A_{i}(\vec{x}), \quad A_{0} & =A_{0}(t) \\
{\left[A_{0}, A_{i}\right] } & =0
\end{aligned}
$$

Such a background corresponds to a vanishing electric field and it was shown that the resulting form of the parity violating effective action, in this background, had an Abelian structure, which was a simple generalization of that for the Abelian case. However, it is generally believed that the Abelian form of this parity violating effective action is a consequence of the above restriction on the background fields.

To analyze the true non-Abelian structure of the parity violating effective action at finite temperature, we have chosen a more direct approach. Namely, we study the amplitudes in perturbation theory, at finite temperature, in the static limit. We choose the static limit for two reasons: i) it is in this limit that large gauge invariance is not manifest in perturbation theory in the Abelian case and, ii) calculations are much more complicated in other limits. We have calculated amplitudes up to the seven point function and our conclusions are as follows. We find that the effective action has two classes of 
terms - one that manifestly depends on $\vec{E}$ and another which does not. In the static limit, we find that the parity violating effective action, which does not manifestly depend on $\vec{E}$ has a form similar to that in [9] (with proper nonAbelian terms). For a vanishing electric field, the non-Abelian structures drop out and our action coincides with that obtained in [9], although our gauge background is more general. We give a path integral derivation of this, for our choice of backgrounds, along the lines of [9] showing that this is indeed the exact parity violating effective action in the static limit, when the electric field vanishes. However, unlike the Abelian case, it is not true that this is the leading order effective action in the static limit. The static parity violating effective action, which manifestly depends on $\vec{E}$, has a family of other terms, which can also contribute at the leading order. Some of these additional terms, in fact, do contribute non trivially at zero temperature and, therefore, can be given a Lorentz invariant description. This is, in fact, completely consistent with the non-Abelian Ward identities of the theory (namely, in the Abelian theory, the Ward identities would imply that the $n$-point function with $n>2$ is, at least, of the order of $p^{n}$ [18]; however, nonAbelian Ward identities do allow dependence on momenta of lower order).

The parity violating static effective action is manifestly invariant under residual static non-Abelian gauge transformations, which are small gauge transformations. In fact, in the strict static limit, there can be no large gauge transformation and, therefore, to analyze the question of large gauge invariance, one has to go away from the static limit. As we have already argued, such a calculation is extremely difficult and, consequently, based on the results of our calculations, we have tried to look for possible generalizations of our results away from the strictly static limit. We derive a possible form of the parity violating effective action, drawing from the studies in the Abelian theory, that will be both small and large gauge invariant. The situation, however, is very different from the Abelian case and it seems to us that many issues remain to be clarified before we understand fully the question of large gauge invariance in such a case.

The paper is organized as follows. In section $\mathbf{2}$, we present our results for the amplitudes, up to the seven point function, in the static limit for terms which do not manifestly depend on $\vec{E}$. We show that these amplitudes can be derived from an action, whose form is similar to that of the parity violating effective action in [9], but with genuine non-Abelian structures. We show that when the electric field vanishes, the non-Abelian interaction terms indeed drop out and our action coincides with that in [9]. We give 
an alternate path integral derivation, showing that this parity violating effective action is exact for the case when the electric field is trivial in this limit. In section 3, we present the amplitudes for the terms which manifestly depend on $\vec{E}$. These amplitudes satisfy the non-Abelian Ward identity following from the residual static gauge invariance of the theory. It is shown that some of these amplitudes do contribute at the same leading order as those in section $\mathbf{2}$ and, therefore, unlike the Abelian case, the effective action that does not manifestly depend on $\vec{E}$, cannot be thought of as the leading order term in the static limit. We present an effective action that would generate these amplitudes. There are two classes of terms in this effective action, i) terms with a nontrivial limit at zero temperature have a covariant form and, ii) those with a vanishing limit at zero temperature have a noncovariant structure. In section $\mathbf{4}$, we try to generalize our results away from the strictly static limit. Following closely to the derivation in the Abelian case, we propose a possible generalization of the parity violating effective action to non-static backgrounds that will be both small and large gauge invariant. We also discuss various issues that remain to be clarified and present a brief conclusion in section $\mathbf{5}$. In appendix $\mathbf{A}$, we compile some useful finite temperature relations. In appendix $\mathbf{B}$, we discuss general properties of thermal gauge transformations and the consequences of a vanishing electric field, both in the Abelian as well as the non-Abelian theory. In appendix $\mathbf{C}$, we discuss briefly, how the restriction in Eq. (1) necessarily leads to an Abelian structure for the parity violating effective action.

\section{Manifestly $\vec{E}$ independent parity violating effective action:}

We are considering $2+1$ dimensional massive fermions interacting with a non-Abelian gauge background described by the Lagrangian density

$$
\mathcal{L}=\bar{\psi}\left(i \gamma^{\mu} D_{\mu}-M\right) \psi
$$

where we assume $M>0$ for simplicity. The fermions are assumed to belong

to the fundamental representation of $S U(N)$ so that the covariant derivative is defined to be

$$
D_{\mu}=\partial_{\mu}+i g A_{\mu}
$$

where the gauge fields, $A_{\mu}$, are matrices belonging to the fundamental representation of $S U(N)$. 
In calculating the amplitudes at finite temperature, we will use the imaginary time formalism, where the time axis is rotated to a finite interval in the imaginary axis (we refer the readers to [15, 19, 20] for details). In this Euclidean space, the three Dirac matrices can be chosen to be anti-Hermitian and a particular representation can be chosen to be (although our results are independent of any choice of the representation)

$$
\gamma_{0}=i \sigma_{3}, \quad \gamma_{1}=i \sigma_{1}, \quad \gamma_{2}=i \sigma_{2}
$$

We are interested in calculating the amplitudes in the static limit, which corresponds to a choice of the background fields of the form

$$
A_{0}=A_{0}(\vec{x}), \quad A_{i}=A_{i}(\vec{x})
$$

without any further restriction on $\vec{E}$.

In this static background, the action has a residual gauge invariance of the form

$$
\begin{aligned}
\psi & \rightarrow U^{-1}(\vec{x}) \psi \\
A_{0} & \rightarrow U^{-1}(\vec{x}) A_{0} U(\vec{x}) \\
A_{i} & \rightarrow U^{-1}(\vec{x}) A_{i} U(\vec{x})-\frac{i}{g} U^{-1}(\vec{x}) \partial_{i} U(\vec{x})
\end{aligned}
$$

As a consequence of this symmetry, it is straightforward to derive that the gauge amplitudes will have to satisfy the Ward identities following from

$$
\partial_{i} \frac{\delta \Gamma_{\mathrm{eff}}}{\delta A_{i}^{a}}+g f^{a b c} A_{0}^{b} \frac{\delta \Gamma_{\mathrm{eff}}}{\delta A_{0}^{c}}+g f^{a b c} A_{i}^{b} \frac{\delta \Gamma_{\mathrm{eff}}}{\delta A_{i}^{c}}=0
$$

where $\Gamma_{\text {eff }}$ is the effective action resulting from evaluating the fermion loops.

As we have already mentioned in the introduction, our calculations of the amplitudes give rise to two classes of effective actions - one that manifestly depends on $\vec{E}$ and another which does not. In this section, we will concern ourselves only with the class that does not depend manifestly on $\vec{E}$. From the structure of the terms which depend manifestly on $\vec{E}$ (which we will discuss in the next section), it is easy to recognize that these may be thought of as resulting from the full effective action when the electric field is covariantly constant. Although the vanishing electric field is a subclass of these configurations, we do not, in fact, assume the electric field to vanish 
and we will comment more extensively on this special subclass later in this section.

The calculation of the amplitudes is tedious, but straightforward, and we will not give details of the calculation which have been described earlier [16, 17] (within the context of an Abelian background). However, let us define some notation to present the results of our calculations in a more manageable form. Let

$$
I^{(r+1)}=\frac{M}{4 \pi r \beta} \sum_{n} \frac{1}{\left(M^{2}+\omega_{n}^{2}\right)^{r}}
$$

where

$$
\omega_{n}=\frac{(2 n+1) \pi}{\beta}
$$

represents the Matsubara frequencies for fermions and $\beta=\frac{1}{T}$ with $T$ representing the temperature. These quantities can all be evaluated in a closed form by successive differentiation of (see appendix $\mathbf{A}$ for the explicit forms of some of the lower order $I^{(r)}$ 's)

$$
I^{(2)}=\frac{1}{8 \pi} \tanh \frac{\beta M}{2}
$$

Let us also define a completely symmetrized fourth rank tensor in the internal space of the form

$$
\Delta^{a b c d}=\delta^{a b} \delta^{c d}+\delta^{a c} \delta^{b d}+\delta^{a d} \delta^{b c}
$$

All the amplitudes can be represented in terms of these quantities in the following way. First, let us note some essential features of the amplitudes in the static limit. In this limit, the parity violating amplitudes involve only an odd number of $A_{0}$ fields. The amplitudes, which lead to the effective action that does not manifestly depend on $\vec{E}$, have the following forms $\left(\vec{k}_{1}, \vec{k}_{2}, \cdots\right.$ correspond to the external momenta associated with the first, second, $\cdots$ indices, all incoming; for the two point function, the momentum is associated with the second index)

$$
\begin{aligned}
\Pi_{0 i}^{a b,(1)}= & -g^{2} \delta^{a b} \epsilon_{i j} k_{j} I^{(2)} \\
\Pi_{0 i j}^{a b c,(1)}= & i g^{3} f^{a b c} I^{(2)} \epsilon_{i j} \\
\Pi_{000 i}^{a b c d,(1)}= & g^{4} \epsilon_{i j} k_{4 j}\left(\frac{2}{N} \Delta^{a b c d}+d^{a b e} d^{c d e}+d^{a c e} d^{b d e}+d^{a d e} d^{b c e}\right) \\
& \times\left(I^{(3)}-2 M^{2} I^{(4)}\right)
\end{aligned}
$$


Here, $f^{a b c}$ and $d^{a b c}$ denote respectively the anti-symmetric and symmetric structure constants for $S U(N)$.

As we go to higher point amplitudes, the calculation involves a color trace over more and more color matrices and as a result, the color factors become more and more complicated. Therefore, we will present the results of our calculations of higher point functions only for $S U(2)$, where we obtain

$$
\begin{aligned}
\Pi_{000 i j}^{a b c d e,(1)} & =-i g^{5} \epsilon_{i j}\left(\delta^{a b} \epsilon^{c d e}+\delta^{a c} \epsilon^{b d e}+\delta^{b c} \epsilon^{a d e}\right)\left(I^{(3)}-2 M^{2} I^{(4)}\right) \\
\Pi_{00000 i}^{a b c d e f,(1)} & =\frac{g^{6}}{2} \epsilon_{i j} k_{6 j} C^{a b c d e f}\left(3 I^{(4)}-16 M^{2} I^{(5)}+16 M^{4} I^{(6)}\right) \\
\Pi_{00000 i j}^{a b c d e f g,(1)} & =-\frac{i g^{7}}{2} \epsilon_{i j} C^{a b c d e f g}\left(3 I^{(4)}-16 M^{2} I^{(5)}+16 M^{4} I^{(6)}\right)
\end{aligned}
$$

where

$$
\begin{aligned}
C^{a b c d e f} & =\sum_{i=1}^{5} C_{i}^{a b c d e f} \\
C_{1}^{a b c d e f} & =\delta^{e f} \Delta^{a b c d}, \quad C_{2}^{a b c d e f}=\delta^{d f} \Delta^{a b c e} \\
C_{3}^{a b c d e f} & =\delta^{c f} \Delta^{a b d e}, \quad C_{4}^{a b c d e f}=\delta^{b f} \Delta^{a c d e}, \quad C_{5}^{a b c d e f}=\delta^{a f} \Delta^{b c d e} \\
C^{a b c d e f g} & =\epsilon^{a f g} \Delta^{b c d e}+\epsilon^{b f g} \Delta^{a c d e}+\epsilon^{c f g} \Delta^{a b d e}+\epsilon^{d f g} \Delta^{a b c e}+\epsilon^{e f g} \Delta^{a b c d}
\end{aligned}
$$

From the definition in Eq. (6), it is easy to see that the parity violating amplitudes, to this order, completely coincide with those following from the action

$$
\Gamma_{\mathrm{eff}}^{\mathrm{PV},(1)}=\frac{i g}{2 \pi} \int d^{2} x \operatorname{Tr} \arctan \left(\tanh \frac{\beta M}{2} \tan \frac{g \beta A_{0}(\vec{x})}{2}\right) B(\vec{x})
$$

where the magnetic field has the standard definition

$$
B=\frac{1}{2} \epsilon_{i j} F_{i j}=\frac{1}{2} \epsilon_{i j}\left(\partial_{i} A_{j}-\partial_{j} A_{i}+i g\left[A_{i}, A_{j}\right]\right)
$$

We also note that the branch of arctan is chosen such that $\Gamma_{\mathrm{eff}}^{\mathrm{PV},(1)}$ is a continuous function of $A_{0}$, which reduces, in the zero temperature limit, to the corresponding CS action.

This is, in fact, the form of the action (but with a non-Abelian structure because the background is more general) derived in [9] for a much more restrictive gauge background. In fact, let us now specialize to the case of 
a vanishing electric field. In a static background, a vanishing electric field would correspond to a field configuration satisfying

$$
-E_{i}=D_{i} A_{0}=\partial_{i} A_{0}+i g\left[A_{i}, A_{0}\right]=0
$$

This would further constrain the relations on the amplitudes following from the Ward identities in Eq. (5). We would like to emphasize that the gauge backgrounds in Eq. (11) and those in Eqs. (3), (15) are inequivalent (although both correspond to $\vec{E}=0$ ) in the sense that there is no smooth gauge transformation which will take one to the other (see appendix $\mathbf{B}$ on more details on the consequences of a vanishing electric field at finite temperature). In this case, it is straightforward to check from Eq. (13) that

$$
\begin{aligned}
\Gamma_{\mathrm{eff}}^{\mathrm{PV},(\vec{E}=0)} & =\frac{i g}{4 \pi} \int d^{2} x \operatorname{Tr} \arctan \left(\tanh \frac{\beta M}{2} \tan \frac{g \beta A_{0}(\vec{x})}{2}\right) \epsilon_{i j}\left(D_{i} A_{j}-\partial_{j} A_{i}\right) \\
& =\frac{i g}{8 \pi} \int d^{2} x \operatorname{Tr} \arctan \left(\tanh \frac{\beta M}{2} \tan \frac{g \beta A_{0}(\vec{x})}{2}\right) \epsilon_{i j}\left(\partial_{i} A_{j}-\partial_{j} A_{i}\right)
\end{aligned}
$$

where the first term vanishes upon integration by parts when the electric field vanishes. This is, in fact, the exact parity violating effective action that was obtained in [9] (We note here that our result, in this limit, differs from that in [9] by a multiplicative factor of $\frac{1}{2}$. However, we do not fully understand if this is a real difference, since these authors imply, in a later publication [12] that when evaluated in a smooth manner, there appears a factor of 2 . So, we will ignore this difference in the multiplicative factor from now on.). It is quite surprising that the perturbative calculations with a less restrictive background seem to yield a parity violating effective action of exactly the same form. In what follows, we will show that this is indeed the exact parity violating effective action, in the static limit, when the electric field vanishes.

Let us consider a fermion interacting with a static background with a vanishing electric field (see Eqs. (3),(15)). In the imaginary time formalism, the action, for such a theory (Fourier transformed in energy), would have the form

$$
\begin{aligned}
S & =\frac{1}{\beta} \sum_{n} \int d^{2} x \bar{\psi}_{n}\left(i \gamma_{i} D_{i}+\gamma_{0}\left(\omega_{n}-g A_{0}(\vec{x})\right)-M\right) \psi_{n} \\
& =\frac{1}{\beta} \sum_{n} \int d^{2} x \bar{\psi}_{n}\left(i \gamma_{i} D_{i}+\gamma_{0} \tilde{\omega}_{n}-M\right) \psi_{n}
\end{aligned}
$$


Here, we have defined

$$
\tilde{\omega}_{n}(\vec{x})=\omega_{n}-g A_{0}(\vec{x})
$$

which is a nontrivial matrix in the internal space, but is proportional to the identity matrix in the Dirac space.

As in the Abelian case, following [8, 9], let us define

$$
\gamma_{0} \tilde{\omega}_{n}(\vec{x})-M=\rho_{n}(\vec{x}) e^{i \gamma_{0} \phi_{n}(\vec{x})}
$$

where

$$
\rho_{n}(\vec{x})=\sqrt{\tilde{\omega}_{n}^{2}(\vec{x})+M^{2}}, \quad \phi_{n}(\vec{x})=\arctan \frac{\tilde{\omega}_{n}(\vec{x})}{M}
$$

Here, $\rho_{n}, \phi_{n}$ are matrices in the internal space, but are proportional to the identity matrix in the Dirac space. Furthermore, since the nontrivial matrix structures for $\rho_{n}(\vec{x}), \phi_{n}(\vec{x})$ arise only from their dependence on $A_{0}(\vec{x})$, it follows (see Eq. (15)) that

$$
\left[\rho_{n}(\vec{x}), \phi_{n}(\vec{x})\right]=0, \quad D_{i} \phi_{n}(\vec{x})=0
$$

We note that this is a crucial difference from the derivation in [9]. The special gauge background in [9] does satisfy this, but here our derivation is quite general.

In terms of these new variables, the action of Eq. (16) takes the form

$$
S=\frac{1}{\beta} \sum_{n} \int d^{2} x \bar{\psi}_{n}\left(i \gamma_{i} D_{i}+\rho_{n}(\vec{x}) e^{i \gamma_{0} \phi_{n}(\vec{x})}\right) \psi_{n}
$$

If we now make a chiral redefinition (from the point of view of two dimensions) of the fermion fields of the form

$$
\psi_{n}=e^{-\frac{i}{2} \gamma_{0} \phi_{n}(\vec{x})} \tilde{\psi}_{n}, \quad \bar{\psi}_{n}=\overline{\tilde{\psi}}_{n} e^{-\frac{i}{2} \gamma_{0} \phi_{n}(\vec{x})}
$$

it is straightforward to show, using the properties of the matrices $\rho_{n}, \phi_{n}$ discussed above as well as Eq. (20), that the action, Eq. (21), takes the form

$$
S=\frac{1}{\beta} \sum_{n} \int d^{2} x \bar{\psi}_{n}\left(i \gamma_{i} D_{i}+\rho_{n}(\vec{x})\right) \tilde{\psi}_{n}
$$

From the definitions in Eq. (19), it is clear that $\rho_{n}$ is parity conserving and, therefore, when functionally integrated, the action in (23) will only contribute to the parity conserving part of the effective action. The parity 
violating part of the effective action will arise only from the Jacobian for the field redefinitions in Eq. (22)

$$
\mathcal{D} \bar{\psi}_{n} \mathcal{D} \psi_{n}=J_{n} \mathcal{D} \overline{\tilde{\psi}}_{n} \mathcal{D} \tilde{\psi}_{n}
$$

which can be calculated following [9, 21] and leads to the parity violating effective action of the form

$$
\begin{aligned}
\Gamma_{\mathrm{eff}}^{\mathrm{PV},(\vec{E}=0)} & =\sum_{n} \log J_{n}=\frac{i g}{4 \pi} \sum_{n} \int d^{2} x \operatorname{Tr} \phi_{n}(\vec{x}) \epsilon_{i j} F_{i j} \\
& =\frac{i g}{8 \pi} \int d^{2} x \operatorname{Tr} \arctan \left(\tanh \frac{\beta M}{2} \tan \frac{g \beta A_{0}(\vec{x})}{2}\right) \epsilon_{i j}\left(\partial_{i} A_{j}-\partial_{j} A_{i}\right)
\end{aligned}
$$

This is, therefore, the exact parity violating effective action in the static case when the electric field vanishes, independent of the directions of the gauge potentials in the internal symmetry space. One can explicitly check that this action leads to the parity violating amplitudes calculated in the static limit (for vanishing electric field) given in Eqs. (10)-(11).

\section{Manifestly $\vec{E}$ dependent parity violating ef- fective action:}

We have also calculated the parity violating amplitudes, up to the seven point function, in the static limit for the general case when there is manifest $\vec{E}$ dependence. For ease of presentation, let us decompose an arbitrary parity violating amplitude as

$$
\Pi_{\mu_{1} \cdots \mu_{n}}^{a_{1} \cdots a_{n}}=\prod_{\mu_{1} \cdots \mu_{n}}^{a_{1} \cdots a_{n},(1)}+\prod_{\mu_{1} \cdots \mu_{n}}^{a_{1} \cdots a_{n},(2)}
$$

Correspondingly, we will also define

$$
\Gamma_{\mathrm{eff}}^{\mathrm{PV}}=\Gamma_{\mathrm{eff}}^{\mathrm{PV},(1)}+\Gamma_{\mathrm{eff}}^{\mathrm{PV},(2)}
$$

where we identify the second class of terms as manifestly depending on $\vec{E}$, namely,

$$
\Pi_{\mu_{1} \cdots \mu_{n}}^{a_{1} \cdots a_{n},(2)}=\prod_{\mu_{1} \cdots \mu_{n}}^{a_{1} \cdots a_{n},(\vec{E})}, \quad \Gamma_{\mathrm{eff}}^{\mathrm{PV},(2)}=\Gamma_{\mathrm{eff}}^{\mathrm{PV},(\vec{E})}
$$

The amplitudes as well as the effective action for manifestly $\vec{E}$ independent terms are already given in the earlier section. Therefore, in this section, we 
will only describe the parts which manifestly depend on $\vec{E}$. Let us also define the following notation for any pair of two-dimensional vectors, $\vec{a}, \vec{b}$, (repeated indices are summed)

$$
\vec{a} \cdot \vec{b}=a_{i} b_{i}, \quad \vec{a} \times \vec{b}=\epsilon_{i j} a_{i} b_{j}
$$

With this, the parity violating amplitudes take the following forms. Since the color factors are not so complicated for the amplitudes up to the four point function, we will give their general forms first,

$$
\begin{aligned}
& \Pi_{0 i}^{a b,(\vec{E})}=-g^{2} \delta^{a b} \epsilon_{i j} k_{j}\left(\frac{k^{2} I^{(3)}}{3}+\frac{\left(k^{2}\right)^{2} I^{(4)}}{10}+\cdots\right) \\
& \Pi_{0 i j}^{a b c,(\vec{E})}=i g^{3} f^{a b c}\left[\frac { I ^ { ( 3 ) } } { 3 } \left\{\delta_{i j} \vec{k}_{2} \times \vec{k}_{3}-\epsilon_{i j}\left(2 k_{2}^{2}+3 \vec{k}_{2} \cdot \vec{k}_{3}+2 k_{3}^{2}\right)\right.\right. \\
& \left.+\left(k_{2 i} k_{2 l}-k_{3 i} k_{3 l}\right) \epsilon_{l j}+\left(k_{2 j} k_{2 l}-k_{3 j} k_{3 l}\right) \epsilon_{l i}\right\} \\
& -\frac{I^{(4)}}{20}\left\{\delta_{i j} \vec{k}_{2} \times \vec{k}_{3}\left(3 k_{2}^{2}+2 \vec{k}_{2} \cdot \vec{k}_{3}+3 k_{3}^{2}\right)\right. \\
& +\epsilon_{i j}\left(6 k_{2}^{4}+5\left(\vec{k}_{2} \cdot \vec{k}_{3}\right)^{2}+15 k_{2}^{2} \vec{k}_{2} \cdot \vec{k}_{3}+5 k_{2}^{2} k_{3}^{2}+k_{2} \leftrightarrow k_{3}\right) \\
& +\left(\epsilon_{i l} k_{3 l}\left(k_{2 j}\left(k_{3}^{2}-k_{2}^{2}\right)+k_{3 j}\left(3 k_{2}^{2}+4 \vec{k}_{2} \cdot \vec{k}_{3}+4 k_{3}^{2}\right)\right)-\left(k_{2} \leftrightarrow k_{3}, i \leftrightarrow j\right)\right) \\
& \left.\left.-\left(\epsilon_{i l} k_{2 l} k_{2 j}\left(4 k_{2}^{2}+4 \vec{k}_{2} \cdot \vec{k}_{3}+3 k_{3}^{2}\right)-\left(k_{2} \leftrightarrow k_{3}, i \leftrightarrow j\right)\right)\right\}+\cdots\right] \\
& \Pi_{000}^{a b c,(\vec{E})}=-i g^{3} f^{a b c} \vec{k}_{1} \times \vec{k}_{2}\left(I^{(3)}+\frac{I^{(4)}}{2}\left(k_{1}^{2}+\vec{k}_{1} \cdot \vec{k}_{2}+k_{2}^{2}\right)+\cdots\right) \\
& \Pi_{000 i}^{a b c d,(\vec{E})}=\frac{g^{4} I^{(3)}}{3} \epsilon_{i j}\left(\left(\frac{2}{N} \delta^{a b} \delta^{c d}+d^{a b e} d^{c d e}\right)\left(3 k_{3}+k_{4}\right)_{j}\right. \\
& \left.+\left(b \leftrightarrow c, k_{2} \leftrightarrow k_{3}\right)+\left(a \leftrightarrow c, k_{1} \leftrightarrow k_{3}\right)+\cdots\right) \\
& \Pi_{0 i j l}^{a b c d,(\vec{E})}=-\frac{g^{4}}{12} I^{(3)} f^{a b e} f^{c d e}\left(\mathcal{A}_{0 i j l}\left(k_{2}, k_{3}, k_{4}\right)-\left(j \leftrightarrow l, k_{3} \leftrightarrow k_{4}\right)+\cdots\right)
\end{aligned}
$$

where $\cdots$ represent higher order terms in momentum and we have defined

$$
\begin{aligned}
\mathcal{A}_{0 i j l}\left(k_{2}, k_{3}, k_{4}\right)= & {\left[\delta_{i j} \epsilon_{l m}\left(k_{2}+k_{4}\right)_{m}-\frac{\delta_{i l} \epsilon_{j m}}{2}\left(k_{2}+2 k_{3}+k_{4}\right)_{m}\right.} \\
& \left.+\epsilon_{i j}\left(k_{2}+2 k_{3}+k_{4}\right)_{l}+\frac{\epsilon_{i l}}{2}\left(k_{2}-k_{4}\right)_{j}+\left(i \leftrightarrow l, k_{2} \leftrightarrow k_{4}\right)\right]
\end{aligned}
$$


The higher point functions are simpler to describe for the case of $S U(2)$ (for simplicity of color factors), where they take the forms

$$
\begin{aligned}
& \Pi_{00000}^{a b c d e,(\vec{E})}=\frac{i g^{5}}{2}\left[( 3 I ^ { ( 4 ) } - 8 M ^ { 2 } I ^ { ( 5 ) } ) \left(\epsilon^{a b e} \delta^{c d} \vec{k}_{1} \times \vec{k}_{2}+\epsilon^{a c e} \delta^{b d} \vec{k}_{1} \times \vec{k}_{3}\right.\right. \\
& +\epsilon^{a d e} \delta^{b c} \vec{k}_{1} \times \vec{k}_{4}+\epsilon^{b c e} \delta^{a d} \vec{k}_{2} \times \vec{k}_{3} \\
& \left.\left.+\epsilon^{b d e} \delta^{a c} \vec{k}_{2} \times \vec{k}_{4}+\epsilon^{c d e} \delta^{a b} \vec{k}_{3} \times \vec{k}_{4}\right)+\cdots\right] \\
& \Pi_{000 i j}^{a b c d e,(\vec{E})}=\frac{2 i g^{5} I^{(3)}}{3} \epsilon_{i j}\left(\delta^{a b} \epsilon^{c d e}+\delta^{a c} \epsilon^{b d e}+\delta^{b c} \epsilon^{a d e}\right)+\cdots \\
& \Pi_{0 i j l m}^{a b c d e,(\vec{E})}=\left[\frac { 2 i g ^ { 5 } I ^ { ( 3 ) } } { 3 } \left(\epsilon^{a b c} \delta^{d e} \epsilon_{i j} \delta_{l m}+\epsilon^{a b d} \delta^{c e} \epsilon_{i l} \delta_{j m}+\epsilon^{a c d} \delta^{b e} \epsilon_{j l} \delta_{i m}\right.\right. \\
& \left.\left.+\epsilon^{a b e} \delta^{c d} \epsilon_{i m} \delta_{j l}+\epsilon^{a c e} \delta^{b d} \epsilon_{j m} \delta_{i l}+\epsilon^{a d e} \delta^{b c} \epsilon_{l m} \delta_{i j}\right)+\cdots\right] \\
& \Pi_{00000 i}^{a b c d e f(\vec{E})}=\left[\frac { g ^ { 6 } ( 3 I ^ { ( 4 ) } - 8 M ^ { 2 } I ^ { ( 5 ) } ) } { 1 0 } \epsilon _ { i j } \left(\left(5 k_{5}+k_{6}\right)_{j} C_{1}^{a b c d e f}\right.\right. \\
& +\left(5 k_{4}+k_{6}\right)_{j} C_{2}^{a b c d e f}+\left(5 k_{3}+k_{6}\right)_{j} C_{3}^{a b c d e f} \\
& \left.\left.+\left(5 k_{2}+k_{6}\right)_{j} C_{4}^{a b c d e f}+\left(5 k_{1}+k_{6}\right)_{j} C_{5}^{a b c d e f}\right)+\cdots\right] \\
& \Pi_{00000 i j}^{a b c d e f g,(\vec{E})}=-\frac{2 i g^{7}}{5}\left(3 I^{(4)}-8 M^{2} I^{(5)}\right) C^{a b c d e f g} \epsilon_{i j}+\cdots
\end{aligned}
$$

where the $C$ 's are defined in Eq. (12).

There are several things to note from the structures of these amplitudes. First of all, we can think of the new structures, $\Pi_{0 i}^{a b,(\vec{E})}$ and $\Pi_{0 i j}^{a b c,(\vec{E})}$, as higher order corrections to the basic structure in Eq. (10). However, structures where all Lorentz indices are "0" or structures with more than two spatial indices are completely new and are not present in terms that do not manifestly depend on $\vec{E}$. Furthermore, even the structures with one and two spatial indices in Eqs. (29)-(30) are of the same order (in powers of momentum) as those in Eqs. ([10)-(11). Thus, unlike in the Abelian case, here the amplitudes, coming from the parity violating effective action that does not manifestly depend on $\vec{E}$, cannot be thought of as leading order contributions in the static limit. Furthermore, it can be easily checked that the amplitudes in Eqs. (29)-(30) satisfy the Ward identity, Eq. (5), following from the residual gauge invariance of the static action.

There is one other significant difference between the structure of the amplitudes in Eqs. (10)-(11) and those in Eqs. (29)-(30), which also reflects the difference in the structure of the Abelian and the non-Abelian theories. 
Namely, it is easy to check that the only amplitudes in Eqs. (10)-(11) which survive in the zero temperature limit are the two and the three point amplitudes. All higher amplitudes in Eqs. (10)-(11) vanish at zero temperature. In contrast, all the amplitudes in Eqs. (29)- (30) have a non-vanishing contribution at zero temperature. Furthermore, we note that all amplitudes with one spatial index (for example, $\Pi_{000 i}^{a b c d,(\vec{E})}$ ) are linear in momentum in the leading order, while those with two spatial indices (for example, $\Pi_{000 i j}^{a b c d e,(\vec{E})}$ ) have a leading behavior which is independent of momentum. In general, we note that all the amplitudes in Eqs. (29)-(30) have a leading momentum dependence which is of lower order than their Abelian counterpart at zero temperature [18] (The Abelian box amplitude, at zero temperature, for example, would have a leading momentum dependence that is at least quartic [16]). This difference in the behavior of the Abelian and the non-Abelian amplitudes is a consequence of the nontrivial form of the Ward identity in the non-Abelian case. In fact, we have explicitly checked that the amplitudes, Eqs. (29)-(30), do satisfy the non-Abelian Ward identities at any temperature.

Given the amplitudes in Eqs. (29)-(30), we can also look for the appropriate action that would give rise to these amplitudes up to this order. With a lot of work, it can be determined that all the leading order terms in the amplitudes in Eqs. (29)-(30) can be derived from the effective action (the normalization is easily determined from the leading order two-point function in Eq. (29))

$$
\Gamma_{1, \mathrm{eff}}^{\mathrm{PV},(\vec{E})}=-\frac{i g^{2} I^{(3)}}{3} \int d^{2} x \operatorname{Tr}\left(E_{i} D_{i} B-B D_{i} E_{i}+\epsilon_{i j} E_{i} D_{0} E_{j}\right) .
$$

In the static limit, of course, the time derivative term in $D_{0}$ gives zero. It is interesting that the relative coefficients between the $\vec{E}-B$ term and the $\vec{E}-\vec{E}$ term could have been different, in principle. The fact that they are the same at any temperature and have a nonzero limit at vanishing temperature, suggests that they come from a single Lorentz invariant structure of the form

$$
\Gamma_{1, \text { eff }}^{\mathrm{PV},(\vec{E})}=-\frac{i g^{2} I^{(3)}}{6} \int d^{2} x \operatorname{Tr} \epsilon_{\mu \nu \lambda} F_{\mu \nu} D_{\alpha} F_{\alpha \lambda}
$$

It is worth pointing out here that there is a second possible Lorentz invariant structure that is available at this order, namely,

$$
\int d^{2} x \operatorname{Tr} \epsilon_{\mu \nu \lambda} F_{\mu \alpha} D_{\nu} F_{\alpha \lambda}
$$


which, however, is related to the structure in Eq. (32) by Bianchi identity. Therefore, at this order, the parity violating effective action has the unique form given in Eq. (32).

In going beyond five point amplitudes (and leading order), there are more possible structures available. We find, after a lot of analysis, that the rest of the structures in the amplitudes in Eqs. (29)-(30) including the seven point functions can be derived from an effective action of the form

$$
\begin{aligned}
& \Gamma_{2, \mathrm{eff}}^{\mathrm{PV},(\vec{E})}=\int d^{2} x \operatorname{Tr}\left[c_{1}^{E B}\left(E_{i} D_{\alpha} D_{\alpha} D_{i} B-B D_{\alpha} D_{\alpha} D_{i} E_{i}\right)\right. \\
& +c_{2}^{E B}\left(E_{i} D_{\alpha} D_{i} D_{\alpha} B-B D_{\alpha} D_{i} D_{\alpha} E_{i}\right) \\
& \left.+\epsilon_{i j}\left(c_{1}^{E E} E_{i} D_{\alpha} D_{\alpha} D_{0} E_{j}+c_{2}^{E E} E_{i} D_{\alpha} D_{0} D_{\alpha} E_{j}\right)\right] \text {. }
\end{aligned}
$$

The four coefficients $c_{1,2}^{E B, E E}$ can all be determined, in principle, comparing the effective action with the diagrammatic results. For instance, the twopoint function in Eq. (29) gives

$$
c_{1}^{E B}+c_{2}^{E B}=-\frac{i g^{2} I^{(4)}}{10},
$$

while the 000 component of the three-point function in Eq. (29) as well as the 00000 component of the five point function in Eq. (30) yield respectively

$$
\begin{aligned}
2 c_{1}^{E E}+c_{2}^{E E} & =-\frac{i g^{2} I^{(4)}}{4} \\
c_{1}^{E E}+c_{2}^{E E} & =\frac{i g^{2}}{20}\left(3 I^{(4)}-8 m^{2} I^{(5)}\right)
\end{aligned}
$$

As a consequence of gauge invariance, all the other results in Eqs. (29) and (30) are consistent with the previous relations (they do not give new relations). A closed system of equations for all the coefficients would require further analysis involving the sub-leading contribution to the $000 i$ component of the four point function. Nonetheless, the coefficients $c_{1,2}^{E E}$ are already fully determined from Eq. (35). It is interesting that, unlike the earlier case of $\Gamma_{1 \text {, eff }}^{\mathrm{PV},(\vec{E})}$, here, $c_{1,2}^{E E}(T) \neq c_{1,2}^{E B}(T)$. Therefore, these terms do not combine to a Lorentz invariant form at finite temperature (Rather, they can be written as the sum of two terms, one of which is manifestly Lorentz invariant while the other is not.). However, it is also clear that, in the limit of zero temperature, when $I^{(5)}=\frac{5}{8 m^{2}} I^{(4)}$ (see appendix B), Eq. (34) and the second relation in 
Eq. (35) are consistent with $c_{1,2}^{E E}(T=0)=c_{1,2}^{E B}(T=0)$, so that the zero temperature effective action that is manifestly Lorentz invariant takes the form

$$
\begin{aligned}
& \Gamma_{2, \text { eff }}^{\mathrm{PV},(\vec{E})}(T=0)=-\frac{i g^{2} I^{(4)}(T=0)}{20} \int d^{2} x \operatorname{Tr} \epsilon_{\mu \nu \lambda}\left(3 F_{\mu \nu} D_{\alpha} D_{\alpha} D_{\beta} F_{\beta \lambda}\right. \\
& \left.-F_{\mu \nu} D_{\alpha} D_{\beta} D_{\alpha} F_{\beta \lambda}\right)
\end{aligned}
$$

Thus, we see that, in the presence of a non-vanishing electric field, the theory develops a family of parity violating effective actions in the static limit at finite temperature of the form

$$
\Gamma_{\mathrm{eff}}^{\mathrm{PV},(\vec{E})}=\Gamma_{1, \mathrm{eff}}^{\mathrm{PV},(\vec{E})}+\Gamma_{2, \mathrm{eff}}^{\mathrm{PV},(\vec{E})}+\cdots
$$

where the higher order terms can be determined from a calculation of perturbative amplitudes at higher orders. These actions are manifestly invariant under the residual gauge transformations in the static gauge. They have a non-vanishing contribution at zero temperature completely consistent with the Ward identities of the non-Abelian theory. In fact, their forms are suggestive and can be trivially extended to the non-static case in which case they will be invariant under a general non-Abelian small gauge transformation.

\section{Going beyond the static limit:}

Our calculations have been strictly in the static limit and have yielded the static effective action of the form

$$
\Gamma_{\mathrm{eff}}^{\mathrm{PV}}=\Gamma_{\mathrm{eff}}^{\mathrm{PV},(1)}+\Gamma_{\mathrm{eff}}^{\mathrm{PV},(\vec{E})}
$$

with the forms of these actions given in the earlier sections. In particular, $\Gamma_{\mathrm{eff}}^{\mathrm{PV},(\vec{E})}$ contains a family of terms involving electric and magnetic fields, which can, in principle, be determined order by order from a calculation of the amplitudes. These actions are invariant under the residual gauge transformations in Eq. (画). However, these are small gauge transformations and our interest has been to understand the behavior of the thermal parity violating effective action under a large gauge transformation. It is clear that, in the strict static limit, there can be no large gauge transformation and we must necessarily go away from the strict static limit if we want to analyze the behavior of the effective action under a large gauge transformation. 
There have been some previous attempts at constructing thermal large gauge transformations [22, 23]. For example, in [22], it has been shown, for the gauge group $S U(2)$, that the gauge transformations

$$
U(t, \vec{x}, \beta)=\exp \left[\frac{2 \pi i t}{\beta} \hat{\theta}(\vec{x}) \cdot \vec{\sigma}\right]
$$

where $\hat{\theta}(\vec{x})$ is a two dimensional instanton, lead to a non-vanishing winding number which is even. Although the general structure of thermal, nonAbelian large gauge transformations is not yet fully understood, it is clear that these must be time-dependent non-Abelian transformations, which are periodic in time with a period $\beta$. We note from our discussion in the last section that the structure of the terms in $\Gamma_{\text {eff }}^{\mathrm{PV},(\vec{E})}$ is suggestive and we have, in fact, already written these in a form that holds for non-static backgrounds. These terms reduce to the appropriate result in the static limit and are invariant under non-Abelian gauge transformations, small and large. The main question, therefore, is how to generalize $\Gamma_{\mathrm{eff}}^{\mathrm{PV}}{ }^{(1)}$ away from the static limit.

As we have argued in the introduction, it is very hard to go beyond the static limit computationally. For general energies and momenta, this is rather involved, even in the case of the self-energy [16] and the level of complexity increases enormously as we go to higher point functions in a non-Abelian background. As a result, we have taken an alternate approach. Namely, we have the effective action in the static limit and we have tried to look for generalizations, away from the strict static limit, that would reduce in a natural manner to the static result in the appropriate limit and will be gauge invariant, only in the simpler case of a vanishing electric field.

To this end, we take guidance from the earlier works on Abelian gauge backgrounds and follow as closely as possible to the structures that arise there, since after all, the non-Abelian result should yield the Abelian one in the appropriate limit. In this connection, we recall that, in an Abelian background with $\vec{E}=0$, the Bianchi identity implies that the magnetic field is static which allows us to choose a background of the form

$$
A_{0}=A_{0}(t), \quad A_{i}=A_{i}(\vec{x})
$$

Subsequently, one can rotate away the time dependence of the $A_{0}$ field, without affecting the time dependence of the $A_{i}$ field, by a suitable time dependent gauge transformation of the form

$$
A_{\mu} \rightarrow A_{\mu}-\partial_{\mu} \Omega, \quad \Omega(t)=\left(\int_{0}^{t}-\frac{t}{\beta} \int_{0}^{\beta}\right) d t^{\prime} A_{0}\left(t^{\prime}\right)
$$


This leads to the time-independent transformed field, $a_{0}(\vec{x}, \beta)$, given by

$$
a_{0}(\vec{x}, \beta)=\frac{1}{\beta} \int_{0}^{\beta} d t^{\prime} A_{0}\left(t^{\prime}, \vec{x}\right)
$$

which becomes space independent for a vanishing electric field, as mentioned above.

Let us now analyze the corresponding issues in the case of a non-Abelian background. Here, for a vanishing electric field, Bianchi identity only implies that

$$
\partial_{0} B+i g\left[A_{0}, B\right]=D_{0} B=0
$$

Namely, the magnetic field is not necessarily static, but is covariantly constant in time. In a non-static background, in this case, we can again find a time dependent gauge transformation which will rotate away the time dependence in the $A_{0}$ field. Namely, under

$$
A_{\mu} \rightarrow \tilde{A}_{\mu}=U^{-1} A_{\mu} U-\frac{i}{g} U^{-1} \partial_{\mu} U
$$

with

$$
U(t, \vec{x})=\left(P e^{-i g \int_{0}^{t} d t^{\prime} A_{0}\left(t^{\prime}, \vec{x}\right)}\right)\left(P e^{i g \int_{0}^{\beta} d t^{\prime} A_{0}\left(t^{\prime}, \vec{x}\right)}\right)^{\frac{t}{\beta}}
$$

where $P$ stands for the path ordering along the time direction, the time dependence of $A_{0}(t, \vec{x})$ can be transformed away much like in the Abelian case. (We avoid the symbol $T$ for time ordering so as not to create confusion with temperature.) For simplicity, we have taken the path ordering with respect to $t=0$, and assume that $U(t=0, \vec{x})=1$. This, then, leads to the periodicity at finite temperature to correspond to

$$
U(\beta, \vec{x})=U(0, \vec{x})=1
$$

which can be easily checked to hold true.

Such a gauge transformation, which preserves the condition (42), goes over to the Abelian one, Eq. (40), in the appropriate limit, since, in that case, path ordering is trivial. The transformation (44) also brings out an interesting feature of the non-Abelian theory, namely, path ordered quantities [24] do arise in this case in a natural manner.

It is worth noting here, for later purposes, that

$$
\operatorname{Tr}\left[\left(P e^{-i g \int_{0}^{\beta} d t^{\prime} A_{0}\left(t^{\prime}, \vec{x}\right)}\right) B(0, \vec{x})\right]=\operatorname{Tr}\left[\left(P e^{-i g \int_{x_{0}}^{x_{0}+\beta} d t^{\prime} A_{0}\left(t^{\prime}, \vec{x}\right)}\right) B\left(x_{0}, \vec{x}\right)\right]
$$


which can be verified using the cyclicity of trace, periodicity of gauge transformations and the fact that $B$ is covariantly conserved. This relation shows that, although individually the path ordered exponential and the magnetic field pick up a dependence on the reference time $x_{0}$ (with respect to which path ordering is defined), the above combination is, in fact, independent of the choice of the reference time. Furthermore, both the path ordered exponential as well as the magnetic field transform covariantly under a general, periodic non-Abelian gauge transformation, for example,

$$
\left(P e^{-i g \int_{x_{0}}^{x_{0}+\beta} d t^{\prime} A_{0}\left(t^{\prime}, \vec{x}\right)}\right) \rightarrow U^{-1}\left(x_{0}, \vec{x}\right)\left(P e^{-i g \int_{x_{0}}^{x_{0}+\beta} d t^{\prime} A_{0}\left(t^{\prime}, \vec{x}\right)}\right) U\left(x_{0}, \vec{x}\right)
$$

Keeping these properties in mind, let us try to generalize the parity violating effective action, in the absence of electric fields (see Eq. (13) or (25)), to non-static backgrounds. To this end, let us note that the transformed field, $\tilde{A}_{0}\left(x_{0}, \vec{x}, \beta\right)$, which depends on the reference time $x_{0}$, can be shown to be related to the path ordered exponential as

$$
e^{-i g \beta \tilde{A}_{0}\left(x_{0}, \vec{x}, \beta\right)}=\left(P e^{-i g \int_{x_{0}}^{x_{0}+\beta} d t^{\prime} A_{0}\left(t^{\prime}, \vec{x}\right)}\right)
$$

This implies that, in the static limit, when the time ordering is irrelevant, $\tilde{A}_{0}\left(x_{0}, \vec{x}, \beta\right)=A_{0}(\vec{x})$, as expected. Furthermore, in the Abelian case, where the limits of integration can also be translated, Eq. (47) goes over to the relation (41).

The above observations are quite important since they allow us to write a generalization of the parity violating static effective action, Eq. (13) (or (25)) to non-static backgrounds of the form

$$
\begin{aligned}
& \Gamma_{\text {eff }}^{\mathrm{PV},(\vec{E}=0)}=\frac{i g}{2 \pi \beta} \int_{0}^{\beta} d x_{0} \int d^{2} x \\
& \times \operatorname{Tr} \arctan \left(\tanh \frac{\beta M}{2} \tan \frac{g \beta \tilde{A}_{0}\left(x_{0}, \vec{x}, \beta\right)}{2}\right) B\left(x_{0}, \vec{x}\right)
\end{aligned}
$$

We remark here parenthetically that, in view of Eq. (45), the above integrand is actually independent of $x_{0}$ for vanishing electric fields. However, for nonvanishing electric fields, the integrand will depend on the reference point and, in this case, the integration over $x_{0}$ is meaningful. 
Let us also note that

$$
\tan \frac{g \beta \tilde{A}_{0}}{2}=i \frac{1-e^{i g \beta \tilde{A}_{0}}}{1+e^{i g \beta \tilde{A}_{0}}}=i\left[1-2 e^{i g \beta \tilde{A}_{0}}+2\left(e^{i g \beta \tilde{A}_{0}}\right)^{2}+\cdots\right]
$$

Namely, the tangent can be expanded in terms of powers of the path ordered exponential. Furthermore, since the path ordered exponential as well as the magnetic field transform covariantly under a general non-Abelian gauge transformation, the effective action in (48) will be invariant under small gauge transformations. Under a large gauge transformation, this action shifts by $\pi n$ (assuming that the magnetic flux is quantized), where $n$ is an integer depending on the branch of arctan.

Although the effective action in Eq. (48) looks superficially similar to that in Eq. (13) (or (25)), to which it reduces in the static limit, it has, in fact, a distinct character. It is invariant under general periodic non-Abelian gauge transformations and is a functional of $\tilde{A}_{0}\left(x_{0}, \vec{x}, \beta\right)$, where (see Eq. (47))

$$
\tilde{A}_{0}\left(x_{0}, \vec{x}, \beta\right)=\frac{i}{g \beta} \log \left(P e^{-i g \int_{x_{0}}^{x_{0}+\beta} d t^{\prime} A_{0}\left(t^{\prime}, \vec{x}\right)}\right)
$$

This is a non-trivial functional of $A_{0}$, in general, and only in the static limit, does it coincide with $A_{0}(\vec{x})$, as we have mentioned earlier.

\section{Conclusion:}

In this paper, we have derived the parity violating thermal effective action induced by $2+1$ dimensional fermions interacting with a non-Abelian static gauge background from a perturbative calculation of amplitudes up to the seven point function. We have attempted to generalize this result to nonstatic backgrounds in a way that naturally reduces to the static action as well as the one for Abelian backgrounds in the appropriate limits, and which is also small and large gauge invariant. The part of the action, which involves non-vanishing electric fields, $\Gamma_{\mathrm{eff}}^{\mathrm{PV},(\vec{E})}$, contains families of terms which are manifestly gauge invariant. Within each family, there are several terms, which are related, in a derivative expansion [25, 26], by the non-Abelian

Ward identities. The generalization of $\Gamma_{\mathrm{eff}}^{\mathrm{PV},(1)}$ in Eq. (13) (or (25)), for the case of vanishing $\vec{E}$, given by the action (48) seems to be the best that one can do by following the parallel with the Abelian case as much as is possible. 
We conjecture that this may represent a relevant part of the complete parity violating effective action away from the static limit, but not the complete action. The reason why this action cannot represent the complete answer, in the absence of electric fields, can be seen as follows. Let us consider the nonstatic, induced CS action at zero temperature, which, then, can be written as

$$
\Gamma_{\mathrm{eff}}^{\mathrm{PV},(\vec{E}=0)}=\frac{i g^{2}}{4 \pi} \int d^{3} x \operatorname{Tr}\left(A_{0} B-\frac{1}{2} \epsilon_{i j} A_{0} D_{i} A_{j}\right)
$$

Note that the second term in Eq. (51) vanishes identically in the static limit, in view of Eq. (15). Our action (48), for $\vec{E}=0$, therefore, represents a generalization of the first term in Eq. (51), which includes all higher order thermal radiative corrections proportional to the magnetic field. To obtain the complete parity violating effective action in this case, one also needs to determine the corresponding higher order corrections to the second term in Eq. (51). This is a non-trivial open problem that remains to be understood. We would like to note here that, in the Abelian case, the two terms have, in fact, the same structure (with different numerical coefficients), which explains why the generalization of the parity violating effective action in the Abelian case is straightforward.

We would like to thank Profs. G. V. Dunne, S. Okubo and J. C. Taylor for many helpful discussions. This work was supported in part by US DOE Grant No. DE-FG 02-91ER40685 and by CNPq and FAPESP, Brazil.

\section{A Explicit low order forms for $I^{(r+1)}$ :}

The explicit results for Eq. (6) can be related to each other by differentiation in relation to $M^{2}$. Using this simple property and the basic formula

$$
I^{(2)}(T)=\frac{M T}{8 \pi} \sum_{n=-\infty}^{\infty} \frac{1}{\left(M^{2}+\omega_{n}^{2}\right)}=\frac{1}{8 \pi} \tanh \left(\frac{M}{2 T}\right),
$$

we have derived the following results

$$
\begin{aligned}
I^{(3)}(T)= & \frac{1}{32 M T \pi}\left(\frac{T}{M} \tanh \left(\frac{M}{2 T}\right)+\frac{1}{2} \tanh ^{2}\left(\frac{M}{2 T}\right)-\frac{1}{2}\right), \\
I^{(4)}(T)= & \frac{1}{64 M^{3} T \pi}\left[\left(\frac{T}{M}-\frac{M}{6 T}\right) \tanh \left(\frac{M}{2 T}\right)\right. \\
& \left.+\frac{1}{2} \tanh ^{2}\left(\frac{M}{2 T}\right)+\frac{M}{6 T} \tanh ^{3}\left(\frac{M}{2 T}\right)-\frac{1}{2}\right],
\end{aligned}
$$




$$
\begin{aligned}
& I^{(5)}(T)=\frac{1}{512 M^{5} T \pi}\left[\left(\frac{5 T}{M}-\frac{M}{T}\right) \tanh \left(\frac{M}{2 T}\right)\right. \\
& +\left(\frac{5}{2}-\frac{M^{2}}{3 T^{2}}\right) \tanh ^{2}\left(\frac{M}{2 T}\right)+\frac{M}{T} \tanh ^{3}\left(\frac{M}{2 T}\right) \\
& \left.+\frac{M^{2}}{4 T^{2}} \tanh ^{4}\left(\frac{M}{2 T}\right)+\frac{M^{2}}{12 T^{2}}-\frac{5}{2}\right]
\end{aligned}
$$

and

$$
\begin{aligned}
& I^{(6)}(T)=\frac{1}{1024 M^{7} T \pi}\left[\left(\frac{7 T}{M}-\frac{3 M}{2 T}+\frac{M^{3}}{15 T^{3}}\right) \tanh \left(\frac{M}{2 T}\right)\right. \\
& +\left(\frac{7}{2}-\frac{2 M^{2}}{3 T^{2}}\right) \tanh ^{2}\left(\frac{M}{2 T}\right) \\
& +\left(\frac{3 M}{2 T}-\frac{M^{3}}{6 T^{3}}\right) \tanh ^{3}\left(\frac{M}{2 T}\right) \\
& +\frac{M^{2}}{2 T^{2}} \tanh ^{4}\left(\frac{M}{2 T}\right)+\frac{M^{3}}{10 T^{3}} \tanh ^{5}\left(\frac{M}{2 T}\right) \\
& \left.+\frac{M^{2}}{6 T^{2}}-\frac{7}{2}\right] \text {. }
\end{aligned}
$$

\section{B Vanishing electric field at finite tempera- ture:}

In this appendix, we will describe some of the consequences of a vanishing electric field, both in the Abelian as well as the non-Abelian theory, which will also clarify why our choice of backgrounds is inequivalent to those in [9].

First, let us consider the Abelian theory where a vanishing electric field implies

$$
E_{i}=\partial_{0} A_{i}-\partial_{i} A_{0}=0
$$

The Bianchi identity, in this case, leads to

$$
\partial_{0} B=0, \quad B=\frac{1}{2} \epsilon_{i j} F_{i j}
$$

so that the magnetic field is static and determines

$$
A_{i}(\vec{x}, t)=\bar{A}_{i}(\vec{x})+\partial_{i} \alpha(\vec{x}, t)
$$


The vanishing of the electric field condition, then, determines

$$
A_{0}(\vec{x}, t)=\partial_{0} \alpha(\vec{x}, t)
$$

At zero temperature, it is clear that we can make a gauge transformation to set the $A_{0}$ field to zero. For example, since under a gauge transformation,

$$
A_{\mu} \rightarrow A_{\mu}^{\prime}=A_{\mu}-i U^{-1} \partial_{\mu} U
$$

we can choose

$$
U=e^{-i \Omega}
$$

where

$$
\Omega=\alpha(\vec{x}, t)=\int_{0}^{t} d t^{\prime} A_{0}\left(\vec{x}, t^{\prime}\right)
$$

which would yield

$$
A_{0}(\vec{x}, t) \rightarrow 0, \quad A_{i}(\vec{x}, t) \rightarrow \bar{A}_{i}(\vec{x})
$$

In other words, at zero temperature, in the Abelian theory, the same gauge transformation that sets $A_{0}$ to zero also makes $A_{i}$ static.

At finite temperature, however, the gauge fields as well as the gauge transformations have to be periodic. In this case, choosing

$$
\Omega(\vec{x}, t)=\int_{0}^{t} d t^{\prime} A_{0}\left(\vec{x}, t^{\prime}\right)-\frac{t}{\beta} \int_{0}^{\beta} d t A_{0}(\vec{x}, t)
$$

we obtain (only true for vanishing electric fields)

$$
A_{0}(\vec{x}, t) \rightarrow A_{0}^{\prime}=\frac{1}{\beta} \int_{0}^{\beta} d t A_{0}(\vec{x}, t), \quad A_{i}(\vec{x}, t) \rightarrow A_{i}^{\prime}=\bar{A}_{i}(\vec{x})
$$

Thus, in the Abelian theory, even at finite temperature, the same gauge transformation makes $A_{0}$ and $A_{i}$ simultaneously static. However, the scalar potential can no longer be set to zero. Note also that, although $A_{0}^{\prime}$ is seemingly space dependent, it is in fact a constant when the electric field vanishes, since

$$
\partial_{i} A_{0}^{\prime}=\frac{1}{\beta} \int_{0}^{\beta} d t \partial_{i} A_{0}=\frac{1}{\beta} \int_{0}^{\beta} d t \partial_{0} A_{i}=0
$$

because of the periodicity of the fields (and the vanishing electric field). 
Let us next consider a non-Abelian theory. Here, the fields are matrices belonging to some representation of the gauge group and the vanishing electric field condition implies (we will set the coupling to unity)

$$
E_{i}=\partial_{0} A_{i}-\partial_{i} A_{0}+i\left[A_{0}, A_{i}\right]=0
$$

The Bianchi identity, in this case, would imply

$$
D_{0} B=\partial_{0} B+i\left[A_{0}, B\right]=0
$$

where

$$
B=\frac{1}{2} \epsilon_{i j} F_{i j}=\frac{1}{2} \epsilon_{i j}\left(\partial_{i} A_{j}-\partial_{j} A_{i}+i\left[A_{i}, A_{j}\right]\right)
$$

Let us note that, under a non-Abelian gauge transformation,

$$
A_{\mu} \rightarrow A_{\mu}^{\prime}=U^{-1} A_{\mu} U-i U^{-1} \partial_{\mu} U
$$

whereas the field strengths, such as $B$ and $E_{i}$, transform covariantly.

We see that, in the non-Abelian theory, a vanishing electric field does not imply that the magnetic field is static, rather it is covariantly static. However, the solution to the covariantly static condition gives

$$
B(\vec{x}, t)=U B(\vec{x}, 0) U^{-1}, \quad U=\left(P e^{-i \int_{0}^{t} d t^{\prime} A_{0}\left(\vec{x}, t^{\prime}\right)}\right)
$$

where $U$ involves a path ordered exponential signifying the non-Abelian nature of the fields. At zero temperature, it is clear that we can make a gauge transformation with $U$ defined above which will make $B$ static, namely,

$$
B(\vec{x}, t) \rightarrow U^{-1} B(\vec{x}, t) U=B(\vec{x}, 0)
$$

It is also easy to check that, under the same gauge transformation,

$$
\begin{aligned}
& A_{0} \rightarrow A_{0}^{\prime}=U^{-1} A_{0} U-i U^{-1} \partial_{0} U=0 \\
& A_{i} \rightarrow A_{i}^{\prime}=U^{-1} A_{i} U-i U^{-1} \partial_{i} U
\end{aligned}
$$

where

$$
\partial_{0} A_{i}^{\prime}=0
$$

when the electric field vanishes. Namely, at zero temperature, the same transformation that makes the magnetic field static, also makes $A_{i}^{\prime}$ static and 
$A_{0}^{\prime}=0$ for a vanishing electric field. It trivially follows now that (because $\left.A_{0}^{\prime}=0\right)$

$$
\left[A_{0}^{\prime}, A_{i}^{\prime}\right]=0
$$

At finite temperature, however, the gauge transformations have to be periodic. We can generalize the earlier gauge transformation to be periodic by defining

$$
U^{(\beta)}=\left(P e^{-i \int_{0}^{t} d t^{\prime} A_{0}\left(\vec{x}, t^{\prime}\right)}\right) e^{i t \tilde{A}_{0}(\vec{x})}
$$

where, periodicity determines

$$
e^{-i \beta \tilde{A}_{0}(\vec{x})}=\left(P e^{-i \int_{0}^{\beta} d t A_{0}(\vec{x}, t)}\right)
$$

Under such a gauge transformation,

$$
B(\vec{x}, t) \rightarrow\left(U^{(\beta)}\right)^{-1} B(\vec{x}, t) U^{(\beta)}=B(\vec{x}, 0)
$$

which follows from the fact that, at finite temperature, the magnetic field has to be periodic, which in turn implies that

$$
\left[A_{0}(\vec{x}, t), B(\vec{x}, 0)\right]=0
$$

Namely, even at finite temperature, the magnetic field is static when the electric field vanishes. In fact, this is a very general feature at finite temperature, namely, a variable that is periodic in the time variable and transforms covariantly under a gauge transformation can be made static, if it is covariantly static.

Under the gauge transformation $U^{(\beta)}$, we have

$$
\begin{aligned}
& A_{0}(\vec{x}, t) \rightarrow A_{0}^{\prime}=\left(U^{(\beta)}\right)^{-1} A_{0} U^{(\beta)}-i\left(U^{(\beta)}\right)^{-1} \partial_{0} U^{(\beta)}=\tilde{A}_{0}(\vec{x}) \\
& A_{i}(\vec{x}, t) \rightarrow A_{i}^{\prime}=\left(U^{(\beta)}\right)^{-1} A_{i} U^{(\beta)}-i\left(U^{(\beta)}\right)^{-1} \partial_{i} U^{(\beta)}
\end{aligned}
$$

Note that under this transformation, while $A_{0}^{\prime}$ becomes static, there is no $a$ priori reason for $A_{i}^{\prime}$ to be static. Let us pursue this question a little bit more in detail.

The vanishing electric field condition, in terms of the new fields, reads (electric field transforms covariantly)

$$
\begin{aligned}
D_{0}^{\prime} A_{i}^{\prime} & \equiv \partial_{0} A_{i}^{\prime}+i\left[A_{0}^{\prime}, A_{i}^{\prime}\right]=\partial_{i} A_{0}^{\prime} \\
\text { or, } \quad \tilde{D}_{0} A_{i}^{\prime} & =\partial_{i} \tilde{A}_{0}
\end{aligned}
$$


We note that, since $A_{i}^{\prime}$ is not covariantly static, it is not a priori clear that it will be static even when we impose periodicity at finite temperature.

In fact, we can solve the above equation exactly and the general solution has the form

$$
A_{i}^{\prime}(\vec{x}, t)=e^{-i t \tilde{A}_{0}}\left[A_{i}^{\prime}(\vec{x}, 0)+\int_{0}^{t} d t^{\prime} e^{i t^{\prime} \tilde{A}_{0}}\left(\partial_{i} \tilde{A}_{0}\right) e^{-i t^{\prime} \tilde{A}_{0}}\right] e^{i t \tilde{A}_{0}}
$$

It is clear from this that, if

$$
\left[\tilde{A}_{0}(\vec{x}), A_{i}^{\prime}(\vec{x}, 0)\right]=0, \quad \text { and } \quad\left[\tilde{A}_{0}(\vec{x}),\left(\partial_{i} \tilde{A}_{0}(\vec{x})\right]=0\right.
$$

then, using periodicity of the fields, we can conclude that $A_{i}^{\prime}$ is static. Let us recall that we still have the freedom of doing a time-independent gauge transformation. However, it is hard to imagine that a single gauge transformation can achieve both these conditions simultaneously. In fact, let us show next that this cannot be achieved unless some further condition is imposed.

Let us define

$$
\left[\tilde{A}_{0},\left(\partial_{i} \tilde{A}_{0}\right)\right]=M_{i}(\vec{x}), \quad\left[\tilde{A}_{0}, A_{i}^{\prime}(\vec{x}, 0)\right]=N_{i}(\vec{x})
$$

If we now make a gauge transformation $\bar{U}(\vec{x})$, then,

$$
\bar{A}_{0}=\bar{U}^{-1} \tilde{A}_{0} \bar{U}, \quad \bar{A}_{i}=\bar{U}^{-1} A_{i}^{\prime} \bar{U}-i \bar{U}^{-1} \partial_{i} \bar{U}
$$

It is straightforward to check that such a transformation can achieve the first of the conditions provided

$$
\left[\left[\tilde{A}_{0},\left(\partial_{i} \bar{U}\right) \bar{U}^{-1}\right], \tilde{A}_{0}\right]=-i M_{i}(\vec{x})
$$

while, for the second, we need

$$
\left[\tilde{A}_{0},\left(\partial_{i} \bar{U}\right) \bar{U}^{-1}\right]=i N_{i}(\vec{x})
$$

The two conditions can, then, be shown to be compatible provided

$$
\left[\tilde{A}_{0}, \partial_{i} \tilde{A}_{0}+i\left[A_{i}^{\prime}(\vec{x}, 0), \tilde{A}_{0}\right]\right]=0
$$

In general, however, there is no reason why this should hold and, unlike in the Abelian theory, in a non-Abelian theory at finite temperature, we cannot go to a static configuration, even if the electric field vanishes. 
However, given a vanishing electric field, we can always choose specific backgrounds that will solve this. The static background $A_{0}=A_{0}(\vec{x}), A_{i}=$ $A_{i}(\vec{x})$ with $D_{i} A_{0}=0$ would solve this, as will the background $A_{0}=A_{0}(t), A_{i}=$ $A_{i}(\vec{x})$ with $\left[A_{0}, A_{i}\right]=0$. However, as we have already seen, these are background choices that cannot necessarily be implemented through a gauge transformation. Furthermore, it is not possible to transform the first background to the second by a gauge transformation. This is simply seen by noting that if we have such a gauge transformation, $U$, it must necessarily be time dependent and satisfy

$$
\begin{aligned}
\partial_{i}\left(U^{-1} A_{0} U-i U^{-1} \partial_{0} U\right) & =0 \\
\partial_{0}\left(U^{-1} A_{i} U-i U^{-1} \partial_{i} U\right) & =0 \\
{\left[U^{-1} A_{0} U-i U^{-1} \partial_{0} U, U^{-1} A_{i} U-i U^{-1} \partial_{i} U\right] } & =0
\end{aligned}
$$

These are three independent conditions, and as we have already seen earlier with two conditions, they cannot automatically be satisfied simultaneously. At least, it cannot be done through well behaved and smooth gauge transformations, which can be seen as follows.

Note that, with a vanishing electric field, the parity violating part of the effective action can be written, in the static background, as

$$
\Gamma_{\mathrm{eff}}^{\mathrm{PV},(\vec{E}=0)}=\frac{i g}{4 \pi} \int d^{2} x \operatorname{Tr} \arctan \left(\tanh \frac{\beta M}{2} \tan \frac{g \beta A_{0}(\vec{x})}{2}\right) \epsilon_{i j} \partial_{i} A_{j}(\vec{x})
$$

On the other hand, in the background of [9], this effective action has the form

$$
\Gamma_{\mathrm{eff}}^{\mathrm{PV},(\vec{E}=0)}=\frac{i g}{2 \pi} \int d^{2} x \operatorname{Tr} \arctan \left(\tanh \frac{\beta M}{2} \tan \frac{g\left(\int d t A_{0}(t)\right)}{2}\right) \epsilon_{i j} \partial_{i} A_{j}(\vec{x})
$$

Without worrying about the difference in the overall multiplicative factor, we note that the latter expression is a total derivative (since $A_{0}$ is only a function of $t$ ) and will vanish unless the background configurations are singular. This makes it clear that we cannot go from the static background to the second background through a smooth and regular gauge transformation. 


\section{Abelian nature of PV action in a special gauge background:}

In this appendix, we will point out briefly why the parity violating effective action, restricted to the particular background field configuration of Eq. (11) cannot have a non-Abelian structure. Let us note that the particular background in Eq. (11) leads to a vanishing electric field and, therefore, we are

necessarily talking about $\Gamma_{\mathrm{eff}}^{\mathrm{PV},(\vec{E}=0)}$. As we will argue, it is the last condition

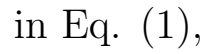

$$
\left[A_{0}, A_{i}\right]=0
$$

which is particularly restrictive and does not allow any non-Abelian structure in $\Gamma_{\text {eff }}^{\mathrm{PV},(\vec{E}=0)}$. In fact, let us first show explicitly that the effective action in [9] contains no non-Abelian structure. When Eq. (93) holds, it follows that

$$
\left[\left(A_{0}\right)^{n}, A_{i}\right]=0
$$

where $n$ is any arbitrary integer. Using this as well as the cyclicity of trace, it follows trivially that

$$
\operatorname{Tr}\left(\left(A_{0}\right)^{n}\left[A_{i}, A_{j}\right]\right)=0
$$

This shows that the non-Abelian terms in the effective action in [9] are, in fact, absent as a consequence of Eq. (93).

Let us now show this in general. We note that Eq. (93) implies one of the following two possibilities.

i) The obvious solution to Eq. (93) is that $A_{0}$ and $A_{i}$ are parallel in the internal space. In this case, the field configurations are truly Abelian. In this case, though, let us note that

$$
\operatorname{Tr} A_{0} A_{i} \neq 0
$$

ii) The second possibility will be to have $A_{0}$ and $A_{i}$ orthogonal in the internal space in a special way. For example, when considering $S U(N)$, we can always choose a basis such that we have $S U(N-1) \oplus U(1)$ embedded in $S U(N)$. In this case, we can choose

$$
A_{0} \in U(1), \quad A_{i} \in S U(N-1)
$$


and they will satisfy Eq. (93). In fact, if $N$ is sufficiently large, we can choose a basis to embed $S U(N-m) \oplus S U(m) \oplus U(1), m>1$ in $S U(N)$. In such a case, we can choose

$$
A_{0} \in S U(m) \oplus U(1), \quad A_{i} \in S U(N-m)
$$

and they will commute. In this case, the gauge field configurations will have truly non-Abelian character. However, since $A_{0}$ and $A_{i}$ belong to orthogonal spaces in this case, we will have

$$
\operatorname{Tr} A_{0} A_{i}=0
$$

As we have already shown in section $\mathbf{2}$ (or as can also be seen in a derivative expansion [25, 26]), in the absence of electric field, the parity violating part of the effective action has the form

$$
\int d^{2} x \operatorname{Tr} F\left(A_{0}\right) B
$$

Such an action will vanish for the second possibility with a truly non-Abelian nature of the gauge field configurations, while it will be nonzero only for the first possibility where the gauge field configurations have an Abelian character. In other words, the last condition in Eq. (四) is too restrictive and necessarily forces the parity violating part of the effective action, in the absence of electric fields, to have only an Abelian structure.

\section{References}

[1] S. S. Chern and J. Simons, Ann. Math. 99, 48 (1974).

[2] S. Deser, R. Jackiw, and S. Templeton, Ann. Phys. 140, 372 (1982).

[3] G. Dunne, Aspects of Chern-Simons Theory, Les Houches Lectures (1998).

[4] K. S. Babu, A. Das, and P. Panigrahi, Phys. Rev. D36, 3725 (1987).

[5] G. Dunne, K. Lee, and C. Lu, Phys. Rev. Lett. 78, 3434 (1997).

[6] S. Deser, L. Griguolo, and D. Seminara, Phys. Rev. Lett. 79, 1976 (1997). 
[7] S. Deser, L. Griguolo, and D. Seminara, Phys. Rev. D57, 7444 (1998); S. Deser, L. Griguolo and D. Seminara, Comm. Math. Phys. 197, 443 (1998).

[8] C. Fosco, G. L. Rossini, and F. A. Schaposnik, Phys. Rev. Lett. 79, 1980 (1997).

[9] C. D. Fosco, G. L. Rossini, and F. A. Schaposnik, Phys. Rev. D56, 6547 (1997).

[10] A. Das and G. Dunne, Phys. Rev. D57, 5023 (1998).

[11] I. Aitchison and C. Fosco, Phys. Rev. D57, 1171 (1998); J. BarcelosNeto and A. Das, Phys. Rev. D58, 085022 (1998); R. Gonzalez Felipe, Phys. Lett. B417, 114 (1998); L. L. Salcedo, Nuc. Phys. B549, 98 (1999); M. Hott and G. Metikas, Phys. Rev. D60, 067703 (1999); S. Feng and D. Zhu, Phys. Rev. D60, 105015 (1999); A. Das, G. Dunne and J. Frenkel, Phys. Lett. 472, 332 (2000).

[12] C. D. Fosco, G. L. Rossini and F. A. Schaposnik, Phys. Rev. D59, 085012 (1999).

[13] H. A. Weldon, Phys. Rev. D47, 594 (1993).

[14] A. Das and M. Hott, Mod. Phys. Lett. A9, 3383 (1994).

[15] A. Das, Finite Temperature Field Theory (World Scientific, NY, 1997).

[16] F. T. Brandt, A. Das, and J. Frenkel, Phys. Rev. D62, 085012 (2000).

[17] F. T. Brandt, A. Das, J. Frenkel, and J. C. Taylor, hep-th/0103221 (to be published in Phys. Rev. D) (2001).

[18] S. Coleman and B. Hill, Phys. Lett. B159, 184 (1985).

[19] J. I. Kapusta, Finite Temperature Field Theory (Cambridge University Press, Cambridge, England, 1989).

[20] M. L. Bellac, Thermal Field Theory (Cambridge University Press, Cambridge, England, 1996).

[21] R. E. Gamboa Saraví, F. A. Schaposnik and J. E. Solomin, Nuc. Phys. B185, 239 (1981). 
[22] R. D. Pisarski, Phys. Rev. D35, 664 (1987).

[23] G. Dunne, private communication.

[24] A. M. Polyakov, Gauge fields and strings (Harwood, Switzerland, 1987).

[25] I. J. R. Aitchison and C. M. Fraser, Phys. Rev. D31, 2605 (1985).

[26] A. Das and A. Karev, Phys. Rev. D36, 623 (1987). 